\title{
Le sable dans les villes du Golfe
}

De la disgrâce à l'infiltration

Sand in the cities of the Gulf

\section{Roman Stadnicki et Manuel Benchetrit}

\section{(2) OpenEdition}

Journals

Édition électronique

URL : https://journals.openedition.org/tc/7329

DOI : $10.4000 /$ tc. 7329

ISSN : 1952-420X

Éditeur

Éditions de l'EHESS

\section{Édition imprimée}

Date de publication : 15 décembre 2013

Pagination : 272-285

ISBN : 978-2-7351-1654-6

ISSN : 0248-6016

\section{Référence électronique}

Roman Stadnicki et Manuel Benchetrit, "Le sable dans les villes du Golfe », Techniques \& Culture [En ligne], 61 | 2013, mis en ligne le 15 décembre 2016, consulté le 29 septembre 2022. URL : http:// journals.openedition.org/tc/7329; DOI : https://doi.org/10.4000/tc.7329 


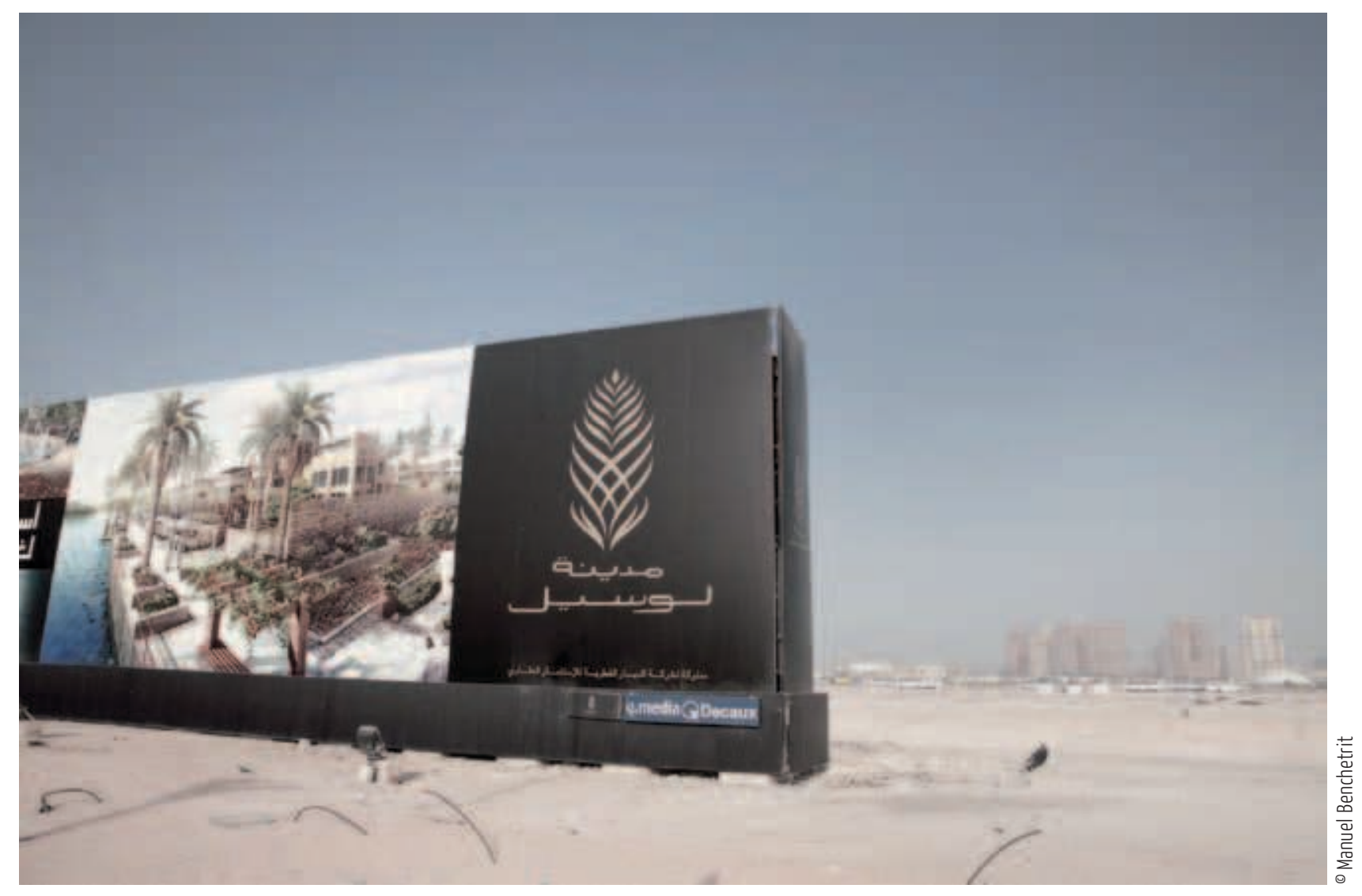


Roman Stadnicki (texte)

Manuel Benchetrit (photographies)

CEDEJ (MAE/CNRS)

roman.stadnicki@cedej-eg.org
Vivre le sable! Corps, matière et sociétés

Techniques E Culture 61, 2013/2: 272-285

\section{LE SABLE DANS LES VILLES DU GOLFE}

\section{De la disgrâce à l'infiltration}

Les villes du Golfe ne cessent de mettre à mal l'ensemble des déterminismes naturels. Il suffit d'en parcourir quelques-unes pour se rendre compte que de l'océan peut naître la terre ferme, que sous l'un des climats les plus brûlants au monde l'air peut être glacial et que du Rub al-Khâlî - désert de sable dont l'austérité fut magnifiée par Wilfried Thesiger (1978) - peuvent surgir les plus solides gratte-ciel. En effet, la conquête illimitée des espaces maritimes par les grands projets touristiques (îles artificielles), la prolifération des centres commerciaux géants et des technopôles climatisés, dont certains hébergent même des pistes de ski couvertes, et, enfin, l'érection de skylines cumulant tous les records ${ }^{1}$, offrent une vue synthétique de l'urbanisation contemporaine - néolibérale dit-on souvent à son sujet - dans les pays du Golfe, mais aussi, peut-être, de ses dérives.

En dépit de cette capacité extraordinaire à s'affranchir des obstacles physiques, les faiseurs de ville locaux (gouvernements et grands groupes de promotion immobilière fonctionnant en synergie) entretiennent un rapport ambivalent avec le cadre naturel - ici, principalement, le désert de sable - dans lequel ils inscrivent leur action. C'est précisément ce que ce numéro Techniques $\&$ Culture nous offre l'opportunité de démontrer, à l'aide de la série photographique qui suit. Étant donné le trop faible matériau discursif recueilli lors de cette mission exploratoire, mais également la rareté des travaux de recherches en sciences sociales consacrés aux villes du Golfe en France et les difficultés d'accès à ces terrains en tant que chercheur et photographe étrangers, qui plus est hors des sentiers battus, ce « documentaire » ne constitue qu'un premier point d'entrée dans la complexité urbaine contemporaine du Golfe arabique. Il prétend néanmoins ouvrir un espace à quelques interrogations clés sur le sujet. 
Montrer au monde industrialisé que les pays du Golfe n'appartiennent pas seulement au désert, aux Bédouins et aux pêcheurs: tel est l'objectif de l'émir Al-Maktoum, lorsqu'il décide de faire de Dubaï une cité-État globale. Le procédé de ce "magnétiseur » (Davis 2007) et des cheikhs voisins très tôt influencés par sa mégalomanie et disposant eux aussi de richesses pétrolifères conséquentes consiste à offrir la moindre portion de désert à l'ingénierie urbanistique d'avant-garde. Au service de la croissance et de la diversification économique, cette ingénierie, qui attire très tôt des architectes de renommée internationale, est soutenue par des campagnes publicitaires hors normes, comme pour annoncer au visiteur que le sable ne sera, ici et là, bientôt plus qu'un vieux souvenir. Au mieux, ce dernier servira à la construction de villas dans les gated communities des périphéries résidentielles; au pire, il sera rendu invisible par l'ornementation urbaine.

Unique ressource locale utilisée dans le développement des villes du Golfe, à tel point qu'il fut parfois nécessaire de l'extraire du fond de la mer ou de l'importer ${ }^{2}$, le sable revient cependant entacher aujourd'hui les images les plus courantes de ces agglomérations, celles de dreamlands high-tech et policés. À l'heure où la crise économique frappe les Émirats du Golfe, le sable s'infiltre sur les zones de chantier dont la livraison est aujourd'hui retardée ou annulée et recouvre certaines infrastructures qui perdent leur utilité tout en conservant leur démesure. Selon certains angles de vue, le sable semble même prendre sa revanche sur l'architecture de verre constitutive du paysage urbain. Il colle aux façades des immeubles comme aux sièges des voitures $4 \times 4$ des usagers, dont l'entretien quasi quotidien constitue une véritable manne financière pour les gérants de laveries automobiles. Les gens du Golfe savent que le sable peut enrayer la dynamique de développement urbain. Certains se souviennent que, dans les années 1960, une tempête de sable a recouvert la crique de Sharjah, située à quelques kilomètres de Dubai, laquelle prenait alors son envol économique. Sharjah a mis de nombreuses années à se remettre de cette catastrophe naturelle.

Ces infiltrations de sable rappellent que le « modèle Dubaï », qui a longtemps prévalu dans le Golfe et qui s'est même bien exporté au-delà de ses frontières, est fragile à plus d'un titre. En raison notamment de l'incapacité de puissantes sociétés immobilières multinationales telles que Emaar et Nakheel à rembourser leurs dettes, un grand nombre de projets urbains inscrits dans la surenchère urbanistico-architecturale ne verront jamais le jour, à l'instar de The Universe, énième groupement d'îles artificielles. Commencée en 2003, la construction de The World - archipel d'îles où figurent les contours des principales terres émergées soidisant visibles depuis la lune -, à l'aide de matériaux provenant des fonds marins aspirés par des dragues, avait déjà largement endommagé l'écosystème. Certaines des îles de ce planisphère de sable sont d'ailleurs toujours inoccupées en 2013, tandis que d'autres sont déjà retournées à la mer. De même, quelle fonction attribuer à la quantité d'espace située au sud de Dubaï et initialement vouée à Dubailand, vaste projet de ville nouvelle et de parc à thème, alors que la plupart des investisseurs se sont aujourd'hui retirés? La gestion de ces quelques « paradis perdus », le recyclage de ces nouveaux déserts artificiels qui ont vidé les émirats de leur ressource première, illustrant les dérives d'un urbanisme néolibéral inspiré des cités-loisirs américaines et fondé sur la privatisation des opérations d'aménagement, constituent aujourd'hui un enjeu urbanistique majeur pour les pays du Golfe.

Il existe un autre enjeu pour l'urbanisation contemporaine dans cette partie du monde: réconcilier la ville avec son environnement désertique, ce qui reviendrait concrètement à « patrimonialiser » le sable, non seulement en construisant de toutes pièces des pastiches 
de vieilles villes arabo-islamiques faisant la part belle aux héritages culturels ${ }^{3}$, mais aussi en préservant plus franchement l'ensemble des ressources naturelles.

Enfin et en guise de conclusion, les gouvernements du Golfe, secoués eux aussi par le « printemps arabe », ne pourront plus longtemps occulter un certain nombre de difficultés sociales, principalement subies par les travailleurs immigrés qui manifestent sporadiquement depuis le milieu des années 2000 pour l'amélioration de leurs conditions de vie et de travail, mais aussi de plus en plus par certains natifs des pays du Golfe: jeunesse urbaine en perte de repères, augmentation du chômage, crise de l'État-providence, exclusion de la nationalité de certaines populations bédouines, etc. Les gouvernements devront tôt ou tard faire face à la prolifération de l'habitat non réglementaire dans les marges urbaines, lesquelles n'ont jamais eu autant d'emprise dans ces villes du Golfe traversées par la crise. La construction de murs autour de quartiers de formation populaire, stratégie d'invisibilisation bien en vigueur dans le Monde arabe en général, ne suffira pas à contenir la révolte qui monte au sein des couches les moins favorisées de la population urbaine. Les manifestations du printemps 2011 survenues notamment dans les grandes villes omanaises qui se sont soldées par la violation des centres commerciaux, des bâtiments publics imposants, des infrastructures routières et autres symboles de l'urbanité locale, apparaissent en effet comme un signe supplémentaire d'épuisement des modes d'urbanisation autoritaristes et inégalitaires (Stadnicki 2012).

\section{Panneau publicitaire planté dans le sable présentant le projet de construction d'un complexe hôtelier de luxe à Dubaï}

Le panneau semble annoncer

la naissance imminente de morceaux de ville du sable lui-même; sable qui disparaît totalement des images de synthèse créées par les acteurs du marketing urbain.

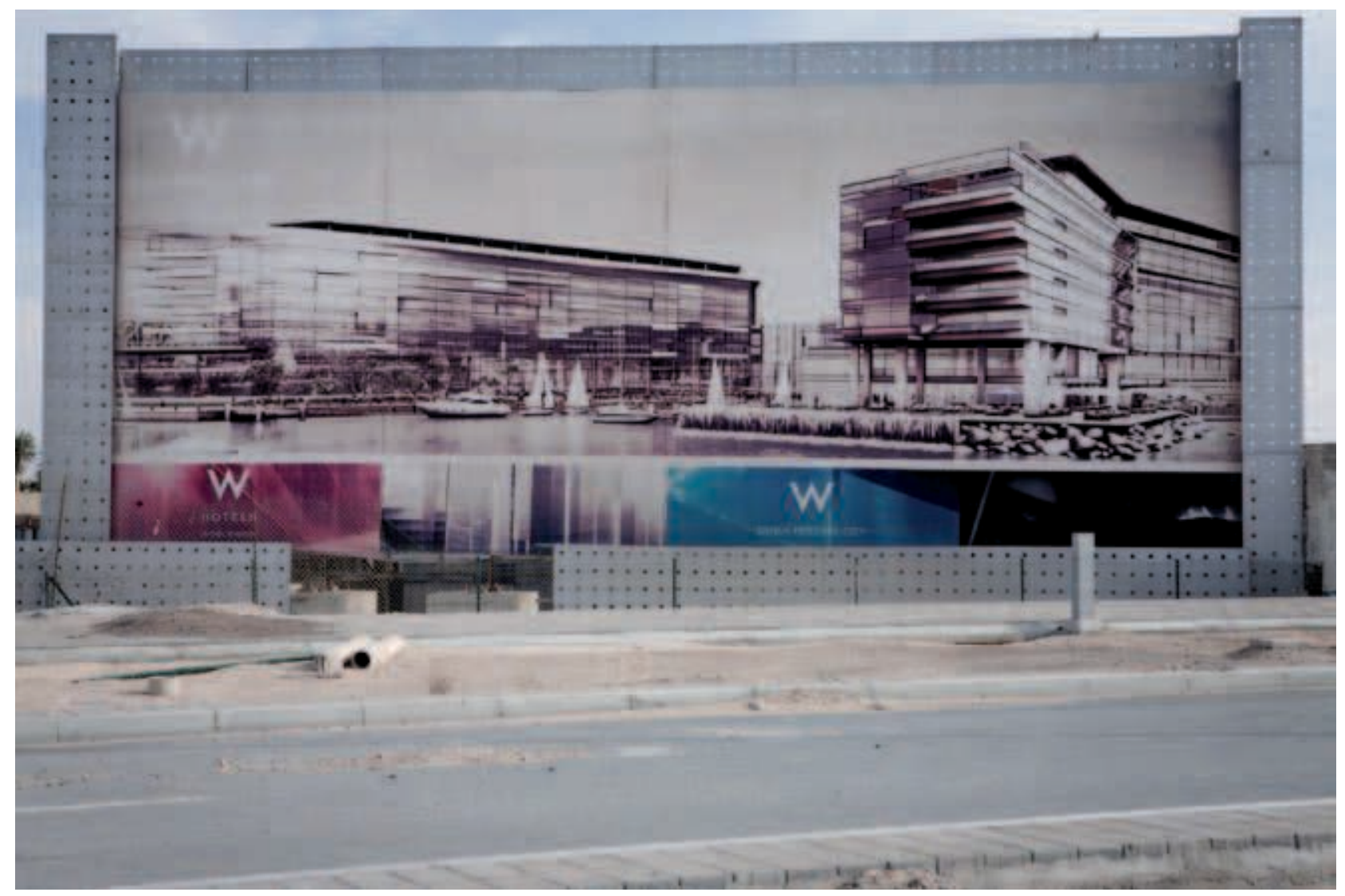


Tours jumelles situées à l'entrée de la future ville nouvelle de Lusaill

au Qatar

Urbanité-mirage où les tours inhabitées semblent zigzaguer, où les routes inutilisées semblent mener nulle part. Le sable apparaît ici comme l'unique habitant de ce paysage urbain presque irréel, balayant toute autre forme de vie sur son passage.

\section{Villas d'une Gated Community} de la banlieue Ouest de Doha

Dans ce secteur, le compound est la principale typo-morphologie urbanistique, révélant des modes

d'habiter auto-exclusifs, fondés sur la fermeture résidentielle, l'entresoi et l'usage indispensable de l'automobile. Au pied du mur protecteur, le sable, qui sert aux constructions le faisant disparaître.

Simple résidu de chantier ou manifestation de la persistance de l'élément naturel?
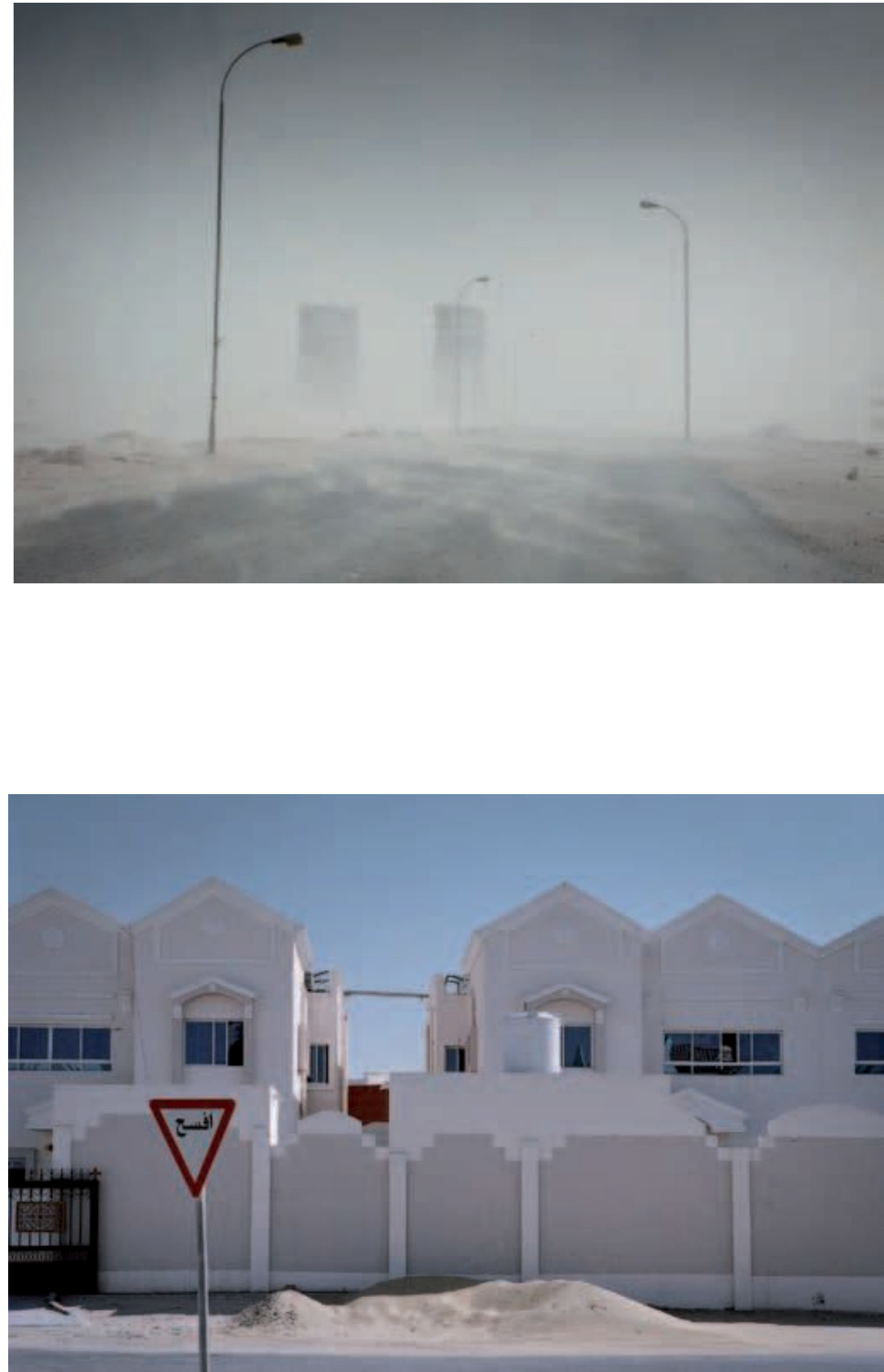


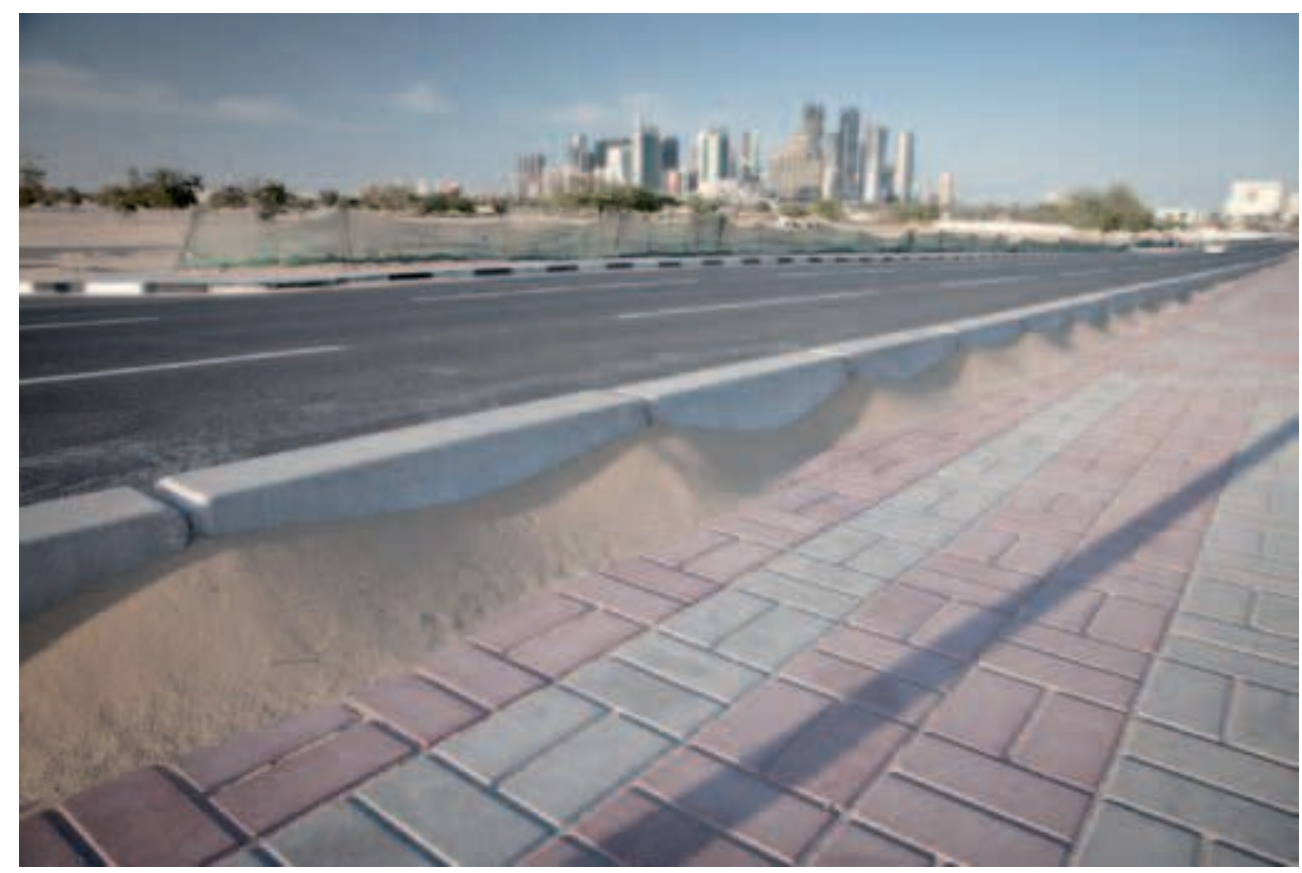

Al-Diwan Street, route menant au centre de Doha.

Nouvelle illustration de la rémanence de la matière

En contrepoint, la skyline, formation urbaine archétypique du Golfe, est érigée comme une oasis. Asphaltage de la ville défiée par le sable volatile, entre solide et liquide, recouvrant, indomptable, insaisissable. Sans cesse repoussé par l'urbanisation

et le tracé des infrastructures,

le sable s'agglutine au ban de la ville et attend la prochaine rafale de vent pour reprendre ses droits.. et ses quartiers.

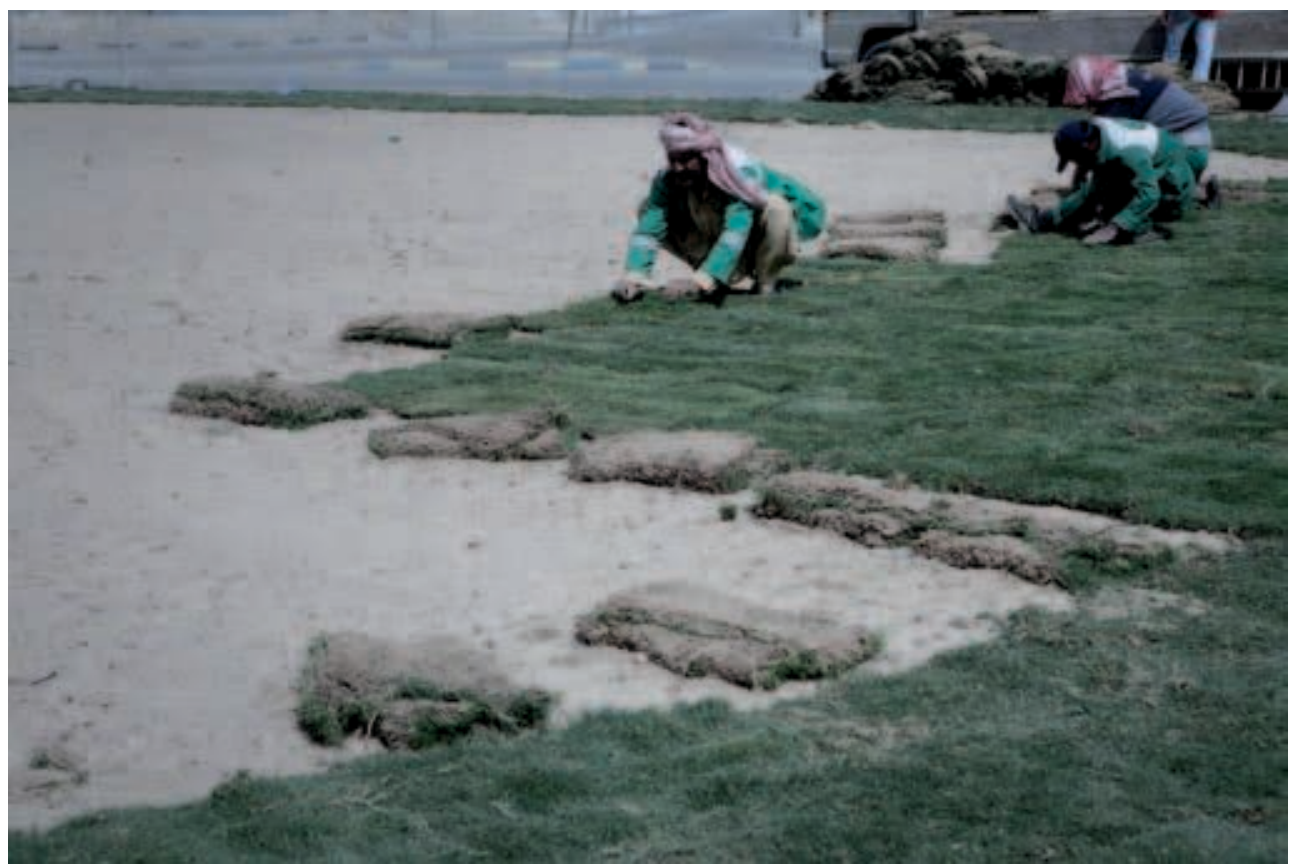

Rond-point menant au Qatar International Exhibition Center, à l'Ouest de Doha.

Pose d'un tapis de gazon importé

Entreprise de végétalisation des infrastructures urbaines qui révèle l'absence de socle environnemental dans les villes du Golfe. Les employés municipaux cherchent à faire disparaître le moindre grain de sable, comme on dissimule la poussière sous le paillasson, sans se soucier du gaspillage d'une autre ressource, l'eau, qu'il faudra utiliser en grande quantité pour que cette pelouse ne devienne jamais jaune, tel le sable qu'elle recouvre. 


\section{Vues ensablées de la lagune de Ras al-Khaimah \\ aux Émirats Arabes Unis et du métro de Dubai inauguré \\ en 2010}

Le sable vient ternir les visions idéalisées des sites d'expansion urbaine potentielle

et des infrastructures modernes.

Ces images donnent à voir l'envers d'un décor aseptisé où s'infiltre le sable contre lequel même l'ingénierie urbanistique la plus puissante ne peut pas lutter.

La vitre devient sale. Le verre redevient sable. La ville ne protège plus de cet élément persistant.
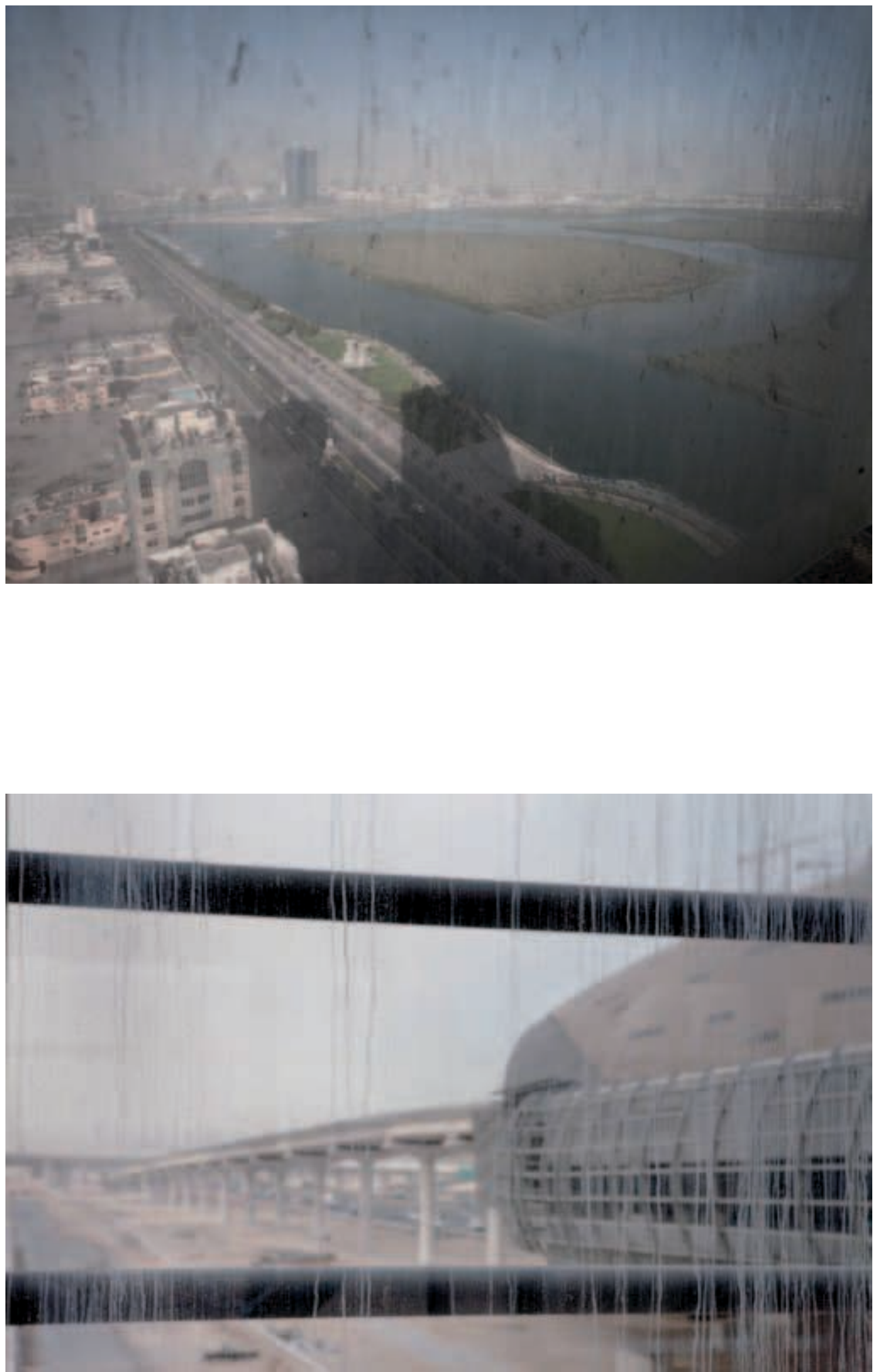


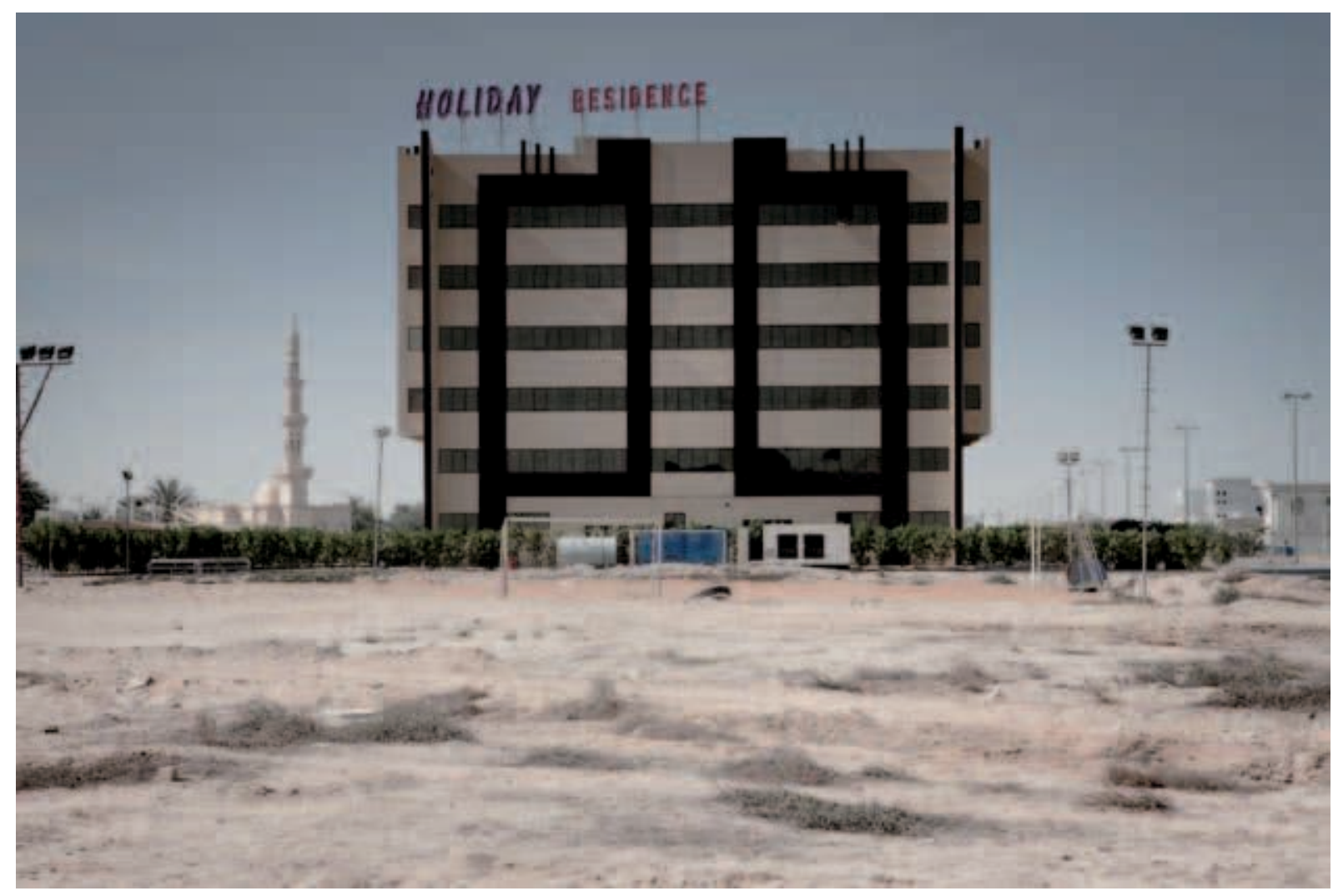

Terrain vague transformé en terrain de foot devant un hôtel de Ras al-Khaimah, Émirats Arabes Unis

La vue offerte aux clients du Holiday Residence semble peu conforme au marketing urbain officiel.

Le cœur même de la ville contient encore des champs de sable formant de vagues étendues. La réalité des villes du Golfe est un paysage urbain à trous fait de territoires inachevés et/ou délaissés. 


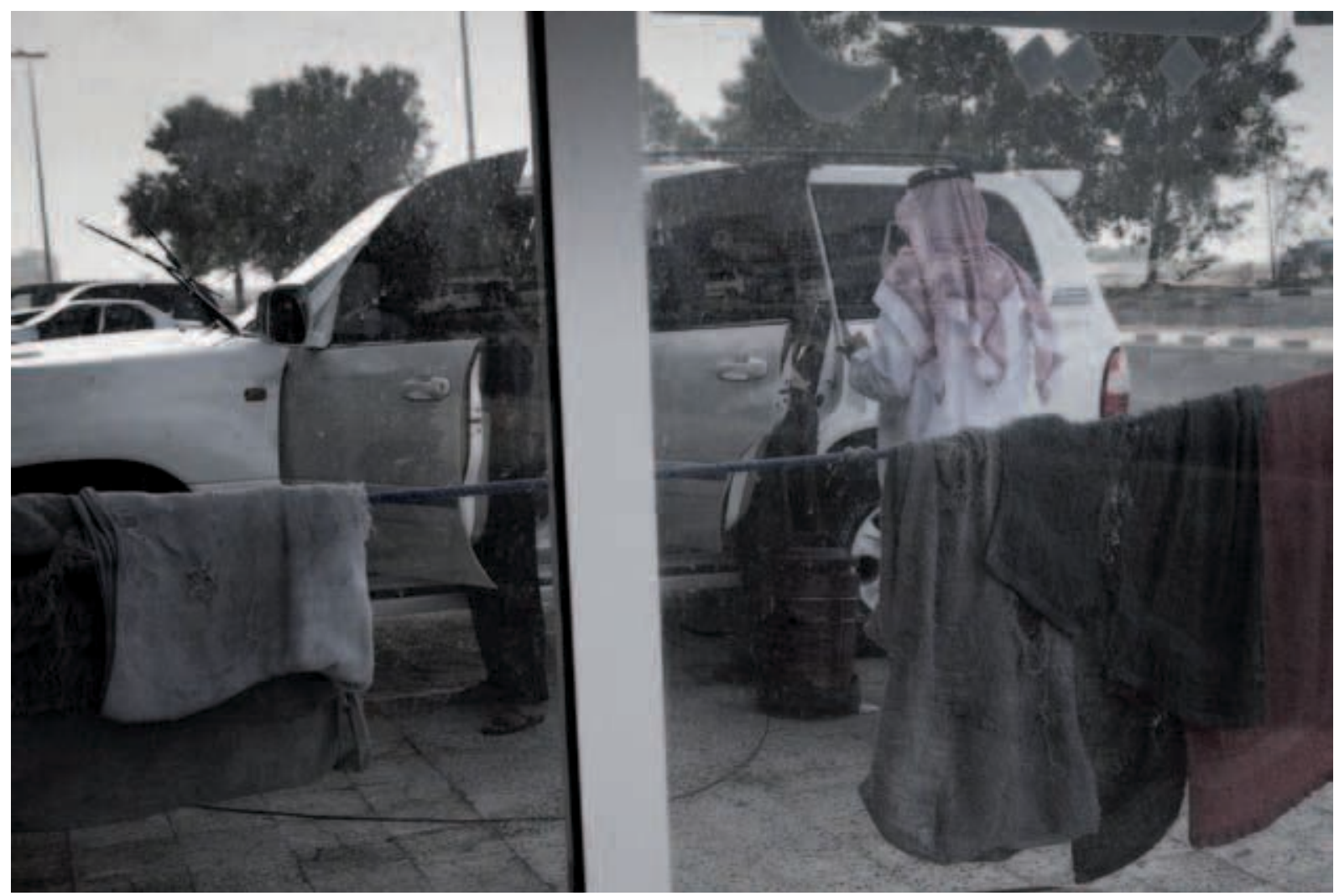

Nettoyage en profondeur, appelé car lifting dans le Golfe, d’un 4x4 Land Cruiser dans une banlieue de Dubaï Scène du petit combat ordinaire que mènent les citadins avec le sable, imposé autant par l'abondance de poussières urbaines que par la norme sociale dans laquelle la voiture joue un rôle prépondérant. D'autant que les propriétaires négligeant l'entretien régulier de leur véhicule sont passibles d'une amende. Une automobile trop poussiéreuse est désormais indexée au chapitre des « mauvais comportements » dans les villes du Golfe. 


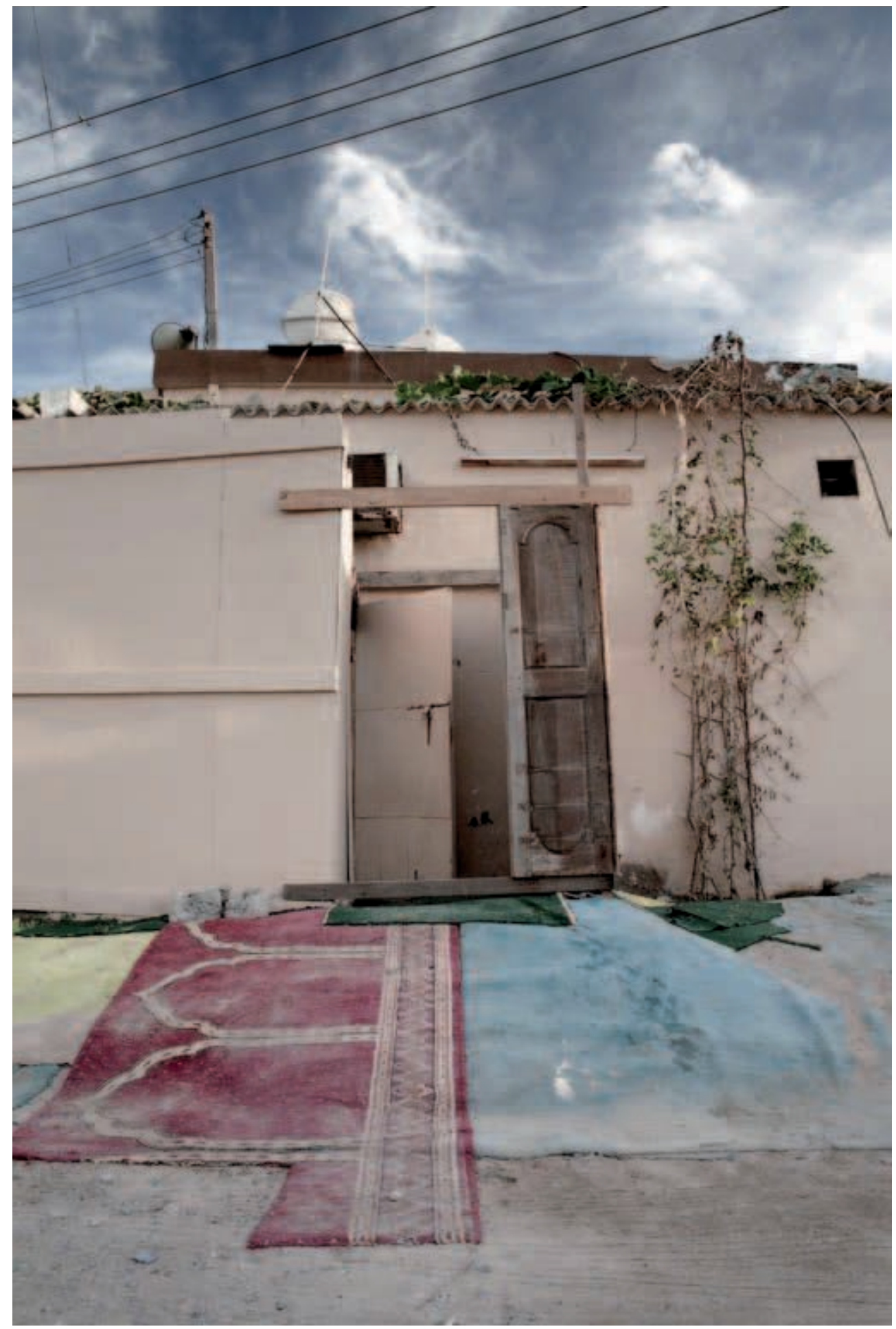

Habitation populaire

à la périphérie de Ras al-Khaimah, Émirats Arabes Unis

Les grands tapis permettent d'empêcher que le sable ne pénètre trop abondamment l'espace intérieur de cette maison, encore plus vulnérable que les tours de verre aux voyages aériens de particules. Mais, l'utilisation de la couleur sable, du matériau bois et du décor végétal produit un lieu plutôt en phase avec l'environnement, qui marche vers la réconciliation entre les hommes et les éléments. 


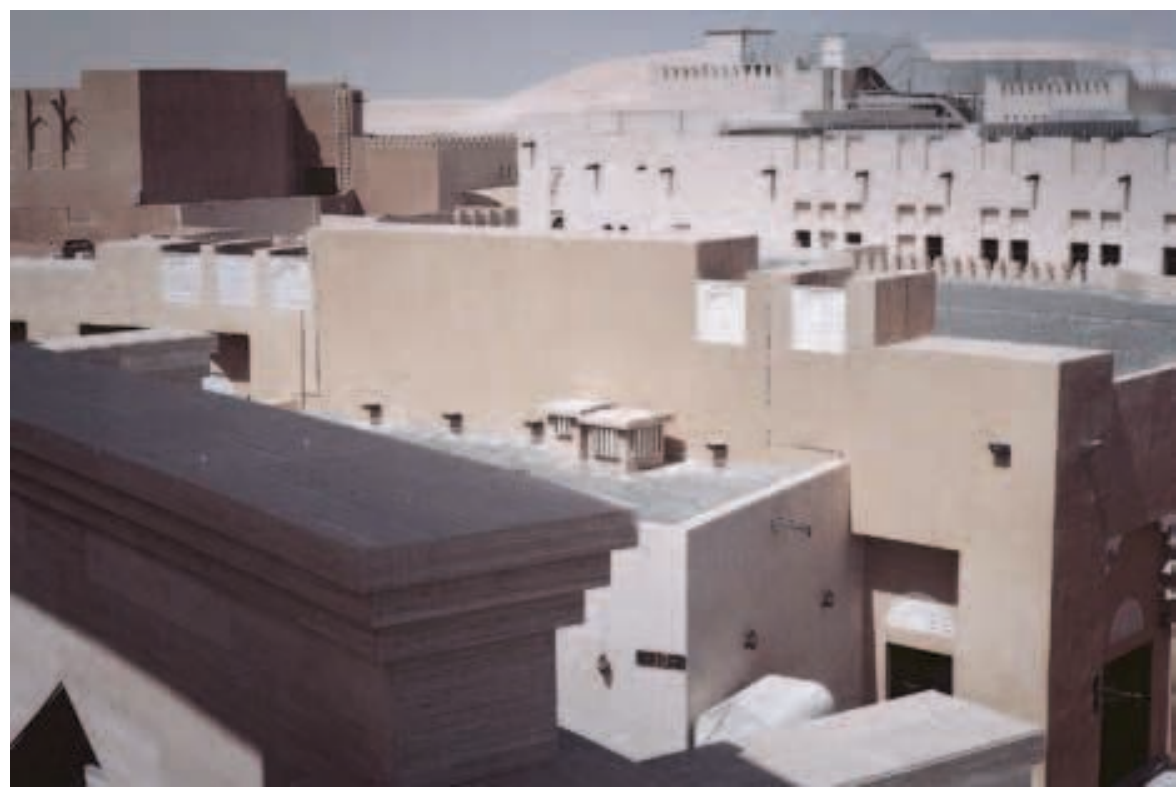

Doha Heritage Village, Qatar

La valorisation du patrimoine semble suivre la même logique que la course effrénée vers la modernité. À Doha, les espaces glorifiant le patrimoine prolifèrent où l'on imite scrupuleusement les formes héritées de temps plus anciens, avec des matériaux modernes et à une allure vertigineuse. Le sable est l'invité d'honneur de ce pastiche de ville ancienne, sa couleur est magnifiée, son grain est figé dans le béton, le trop-plein forme un environnement dunaire en arrière-plan. La ré-injection d'éléments patrimoniaux exhumés par une re-fabrication volontariste signale très certainement le souhait d'endiguer les influences identitaires de l'extérieur par la visibilité urbaine. Ces deux démarches que tout oppose en apparence s'inscrivent dans une dialectique entre imitation et modèle, entre création « in vitro » et « plus vraie que nature », entre juxtaposition et remplacement. 


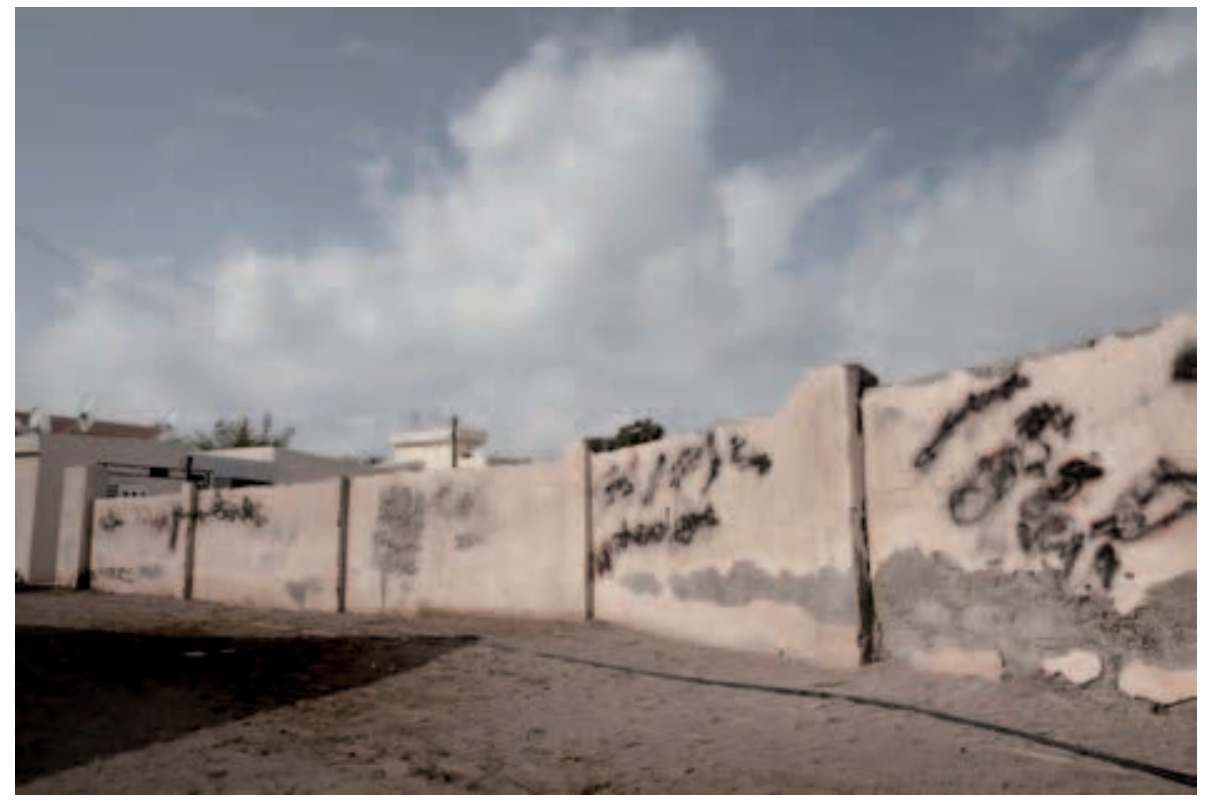

Matérialisation de frontières cadastrales, visant probablement à éviter le déplacement des talus de sable mais aussi à limiter les constructions informelles, dans Majis, quartier côtier de Sohar, Oman

Ironie involontaire, le sable étant utilisé pour la construction, la couleur du mur en reste la même, et l'effet produit s'apparente à une continuité visuelle, plutôt qu'à la rupture initialement prévue. L'apparition inopinée de graffitis à l'heure du « Printemps arabe » résonne comme un écho à l'impuissance d'un interventionnisme qui, en souhaitant contenir le sable, a non seulement échoué mais érige de surcroît le support d'une certaine forme d'expression populaire, probablement non désirée. 


\section{NOTES}

Photo d'ouverture: Panneau publicitaire planté dans le sable présentant le projet de construction d'une ville nouvelle. Toutes les illustrations de l'article sont à mettre au crédit de Manuel Benchetrit.

\section{Démarche :}

* Roman Stadnicki, le géographe, identifiait les sites pertinents avec son angle de recherche, qui concerne les dynamiques sociales et économiques des périphéries urbaines, tout en laissant la possibilité au photographe d'élaborer son propos dans le périmètre établi. Le géographe ne s’interdisait cependant pas de désigner concrètement des images à prendre au fil des entretiens informels qu'il réalisait avec la population ou bien de suggérer de façon plus abstraite la transcription visuelle de concepts déjà élaborés. En ce sens, les photographies pouvaient être tantôt « artistiques » tantôt « documentaires ». De même, le regard géographique du chercheur opérait un glissement nécessaire vers le photographique. La plasticité de cette démarche a donc naturellement appelé un questionnement sur les enjeux du dialogue entre photographie et géographie sociale de l'urbain dans la mise en œuvre d'un tel projet de recherche. ** Pour un aperçu des travaux de M. Benchetrit, le photographe, voir http://aberrances.blogspot.com.
1. Burj Khalifa, inauguré en 2009 à Dubaï, mesure 828 mètres. C'est le plus haut immeuble au monde.

2. Pour faire couvrir ses pistes de courses, le prince héritier de Dubaï a importé 3000 tonnes de sable d'Angleterre en 2006. Quant au sable servant à remplir les trappes du terrain de golf du complexe Dubaï Sports City, il proviendrait d'Arabie Saoudite.

3. Dès le milieu des années 1990, la municipalité d'Abu Dhabi suggère d'introduire des éléments de la culture vernaculaire dans le design des futurs immeubles (Boulanger 2010). Au cours de ces années se multiplient, un peu partout dans la région, les projets de « heritage » ou « cultural villages » faisant la promotion de l'identité bédouine et des constructions traditionnelles (tentes posées sur le sable, maisons en brique de boue, moulins à vent).

\section{REMERCIEMENTS}

Les auteurs souhaitent remercier le New York University Institute à Abu Dhabi, le campus Moyen-Orient de Science-Po à Menton, l'UMR CITERES-EMAM, la Maison des Sciences de l'Homme Val de Loire, le Centre Français d'Archéologie et de Sciences Sociales de Sanaa, l'Ambassade de France en Oman, la Mission archéologique britannique au Qatar, ainsi que Marie Roussel pour le regard qu'elle a porté sur cet essai.

\section{RÉFÉRENCES}

Boulanger, P. 2010 L'identité bédouine dans l'architecture et l'urbanisme à Abu Dhabi au début du xxi siècle, in P. Boulanger \& C. Hullo-Pouyat (dir.), Espaces urbains à l'aube du vingt et unième siècle: patrimoine et héritages culturels. Paris: PUPS : 231-252.

Davis, M. 2007 Le stade Dubaï du capitalisme. Paris: Les prairies ordinaires.

Stadnicki, R. 2012 Enquête géophotographique aux marges des villes du Golfe arabique... Ou comment dépasser la critique, Carnets de géographes 4. [En ligne], mis en ligne le 17 septembre 2012. URL: http:// www.carnetsdegeographes.org/carnets_terrain/terrain_04_04_Stadnicki.php consulté le 22 octobre 2014.

— 2009 Nouvelles centralités et recompositions socio-spatiales dans le Grand Sanaa (Yémen), Thèse, Université de Tours.

Thesiger, W. 1978 Le désert des déserts. Paris: Plon. 


\section{POUR CITER CET ARTICLE}

Stadnicki, R. \& Benchetrit, M. 2014 Le sable dans les villes du Golfe. De la disgrâce à l'infiltration, in S. Boulay \& M.-L. Gélard, Vivre le sable! Corps, matière et sociétés, Techniques \& Culture 61 : 272-285.

\section{RÉSUMÉ}

Le sable dans les villes du Golfe. De la disgrâce à l'infiltration. Cet article, qui revêt la forme d'un documentaire photographique, rend compte des réflexions soulevées par une exploration en péninsule arabique début 2011. L'intention initiale de cette mission était de conjuguer la photographie et la géographie afin d'interroger les identités multiformes des territoires urbains émergents, dans un échantillon de grandes villes de cette sous-région du monde arabe: Sanaa (Yémen), Mascate et Sohar (Oman), Doha (Qatar), ainsi que Dubaï, Ajman, Sharjah et Ras Al Khaimah (Émirats Arabes Unis). Cet itinéraire a été conjointement élaboré par Roman Stadnicki, géographe, qui vit et travaille aujourd'hui au Caire, après avoir passé deux ans à Sanaa (Yémen) dans le cadre de la préparation d'un doctorat (Stadnicki 2009), et Manuel Benchetrit, photographe, qui, après avoir enseigné le français à l'Université de Khartoum (Soudan), a été responsable du Centre Culturel Français de Salalah (Sultanat d'Oman). La série photographique présentée ici questionne les liens entre la ressource sable et l'urbanisme dans cette région du monde arabe.

\section{MOTS-CLÉS}

Sable, urbanisation, crise, Dubaï, photographie

\section{KEYWORDS}

Sand, urbanization, crisis, Dubai, photography 